\title{
Lose 500,000 Creatine Phosphokinase With a 2-Hour Workout: A Case Report on Rhabdomyolysis in a Novice Athlete
}

\author{
Asif Khan ${ }^{\mathrm{a}, \mathrm{b}}$, George Frega ${ }^{\mathrm{a}}$, Elie El-Charabaty ${ }^{\mathrm{a}}$, Suzanne El-Sayegh ${ }^{\mathrm{a}}$
}

\begin{abstract}
The co-occurrence of acute kidney injury secondary to rhabdomyolysis in a young patient can present as a prognostic and therapeutic challenge. Here we report a case with an unusually high creatinine phosphokinase of over 500,000 U/L after a 2-hour workout session at the gym. The challenge was to assess if the need for renal replacement therapy was warranted and whether IV fluid therapy with close monitoring would be enough to treat him, given he had a good urine output and no acid base disorder. The literature is reviewed as an attempt to delineate a rational approach to evaluating novice athletes at risk for rhabdomyolysis.
\end{abstract}

Keywords: Rhabdomyolysis; Acute kidney injury; Myoglobin; Creatinine kinase

\section{Introduction}

Rhabdomyolysis (RB) is a condition that induces a rapid breakdown of skeletal muscle cells, and involves leakage of large quantities of potentially toxic intracellular contents into plasma. It is characterized by marked elevation of creatine phosphokinase (CK), myoglobinuria, and aldolase in blood [1]. The most common symptoms are muscle weakness, myalgia and high colored urine [2]. However, up to $50 \%$ of the cases may present as asymptomatic [1]. There is no established serum CK level cut-off for diagnosing RB, but a CK level > $5,000 \mathrm{IU} / \mathrm{L}$ indicates serious muscle injury [3].

We report a case of an active young male patient who presented to Staten Island University Hospital with severe fatigue and reddish brown urine following $2 \mathrm{~h}$ of intense workout with a CK of over 500,000 IU/L.

Manuscript accepted for publication September 26, 2016

aDepartment of Nephrology, Staten Island University Hospital, 500 Seaview Ave, Suite 225, Staten Island, NY 10305, USA

${ }^{b}$ Corresponding Author: Asif Khan, Department of Nephrology, Staten Island University Hospital, 500 Seaview Ave, Suite 225, Staten Island, NY 10305, USA. Email: asifmk360@gmail.com

doi: http://dx.doi.org/10.14740/wjnu280e

\section{Pathophysiology}

During muscle injury, there is a decreased energy production that impedes the energy-dependent ion pumps, such as $\mathrm{Na}^{+} / \mathrm{K}^{+}$ ATPase and $\mathrm{Ca}^{2+}$ ATPase in the sarcolemma $[4,5]$. This results in an increase cellular permeability to sodium ions $[5,6]$. To counter the sodium influx, the cell increases the activity of the $2 \mathrm{Na}^{+} / \mathrm{Ca}^{2+}$ exchanger, which removes the excess intracellular sodium ions from the cytoplasm by exchanging it with calcium ions, eventually increasing the cytoplasmic calcium $[6,7]$. Additionally, as the concentration of extracellular ionized calcium is 10,000 times higher than that in the intracellular space [8], any interruption of the plasma membrane continuity results in massive influx of extracellular calcium into the cytoplasm driven by the gradient [9].

Due to the cell's incompetent energy-dependent ion pumps and the increased permeability to calcium, the muscle cells get overloaded with calcium ions. Calcium activates a variety of proteolytic enzymes that injure the cell membrane, allowing efflux of cellular components such as potassium, myoglobin, phosphate and CK into the circulation. Additionally, the extracellular calcium is forced to the intracellular space leading to interaction with actin and myosin causing muscle destruction [10-12].

Myoglobin is composed of globin, and a molecule of heme that is found in the muscle tissue of vertebrates, giving its characteristic color. Physiologically, the excretion of myoglobin occurs mainly through the renal system. During RB, excess myoglobin from skeletal muscle cells enters the plasma known as myoglobinemia [7]. When the concentration of myoglobin exceeds the reabsorbing capacity of glomerular cells, an excessive amount of myoglobulin appears in the urine as myoglobinuria that may be detected either with a urine dipstick (microscopic myoglobinuria) or as reddish-brown urine in the case of severe RB [9, 13]. Furthermore, if more than $100 \mathrm{~g}$ of skeletal muscle is damaged, the serum haptoglobin binding capacity becomes saturated with myoglobin and remaining free circulating myoglobin can precipitate and obstruct the renal tubules, leading to tubular necrosis, and development of acute kidney injury (AKI).

About $7-10 \%$ of all cases of AKI in United States are contributable to RB [14]. Chatzizisis et al explored the RB complications that included life-threatening disorders such as hyperkalemic cardiotoxicity, acute renal failure, and early hy- 


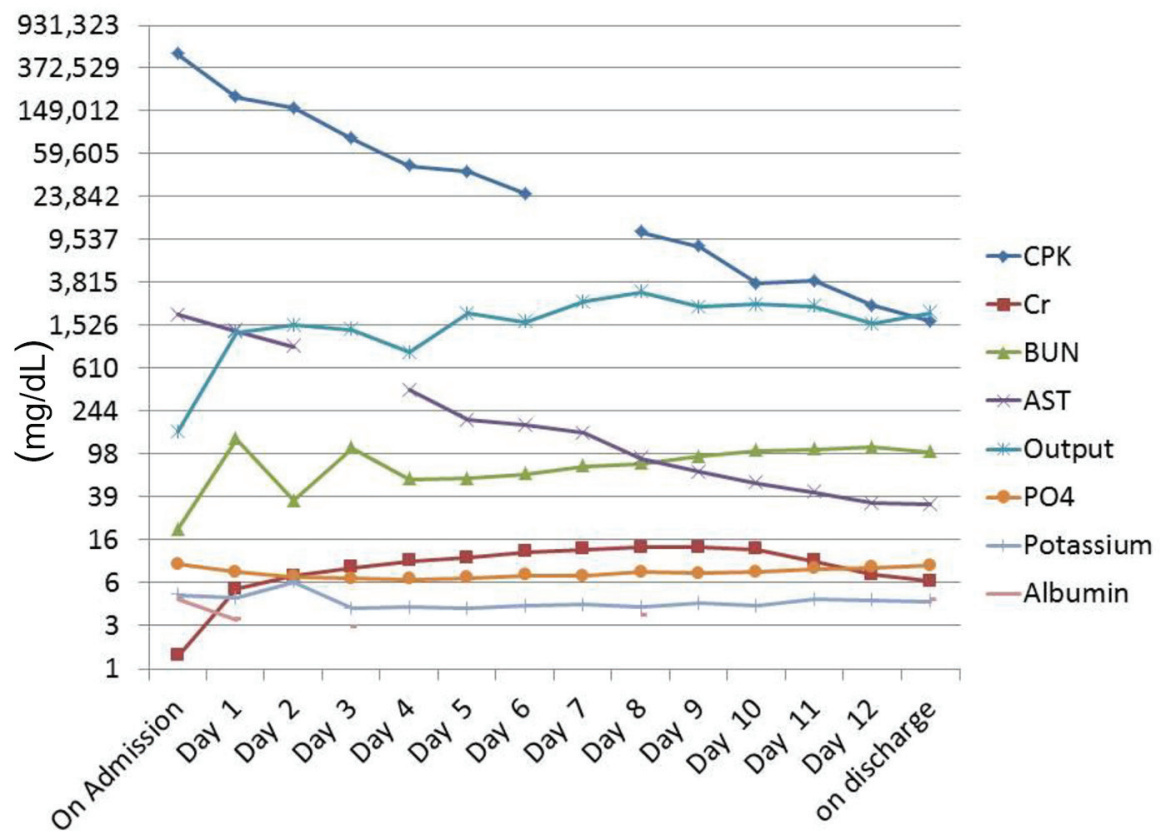

Figure 1. Lab results over the course of treatment.

pocalcemia [15]. Irrespective of the cause of $\mathrm{RB}$, the mortality rate may still be as high as $8 \%$ with an RB-induced AKI mortality rate of approximately $20 \%$ [16].

\section{Case Report}

A 20-year-old young male with no significant past medical history presented to the emergency room with severe fatigue and pain all over his body with reddish brown urine following $2 \mathrm{~h}$ of intense workout at the gym. He was well hydrated during his workout session. He denied any history of trauma, chest pain, breathlessness or use of illicit drugs.

He lives an active lifestyle and engages in school sports like football and basketball on regular basis. He occasionally takes aspirin for body pain and aches. Two days prior to admission, he spent $2 \mathrm{~h}$ at the gym specifically doing upper body workout, carrying 35 - 75 Ibs. He has not been to the gym for the last 5 years. On the first day, he tolerated the workout well; however, on the second day after working out for an hour in the gym, he immediately felt exhausted and fatigued, but forced himself to continue for another hour. He was not on any workout supplements or weight loss products. After his workout session, he experienced intense diffuse muscle pain, which restricted his movement. He took two tablets of Aleve that mildly alleviated the pain. Four hours later, he started to pass reddish brown urine and was brought to the emergency department.

Vitals on presentation revealed normal heart rate of 118 beats per minute, blood pressure of $126 / 78 \mathrm{~mm} \mathrm{Hg}$, and respiratory rate of 20 breaths per minute and he was afebrile. On examination, he was well oriented to time, place and person. Lung and heart examinations were normal. Abdominal was soft and non-tender. Musculoskeletal system examination re- vealed muscle pain with a severity of $8 / 10$ and tenderness in arms, back and shoulders with intact reflexes.

His initial set of ER lab showed an elevated hemoglobin of $16.7 \mathrm{~g} / \mathrm{dL}$ with a white cell count of $13,440 / \mathrm{mm}^{3}$. The CK level on admission was about 510,924 IU/L. Creatinine $(\mathrm{Cr})$ was $1.33 \mathrm{mg} / \mathrm{dL}$ with blood urea nitrogen (BUN) of $20 \mathrm{mg} / \mathrm{dL}$, AST was 1,951 IU/L, ALT was $381 \mathrm{IU} / \mathrm{L}$, anion gap was 11.0 $\mathrm{mEq} / \mathrm{L}$, albumin was $4.5 \mathrm{~g} / \mathrm{dL}$, and inorganic phosphate was 5 $\mathrm{mg} / \mathrm{dL}$. His urine analysis showed specific gravity $>1.030$ and protein $>300 \mathrm{mg} / \mathrm{dL}$, blood and bilirubin was detected as high, and nitrite was positive with moderate leukocytes. RBCs casts were present with mucous threads and the urine myogloblin level $>12,700 \mu \mathrm{g} / \mathrm{L}$ (reference range: $<28 \mu \mathrm{g} / \mathrm{L}$ ). Drug screen turned out to be negative.

An initial diagnosis of RB was made and the patient was bolused on $3 \mathrm{~L}$ of sodium chloride $0.9 \%$. Muscle aches were managed by I/V morphine $2 \mathrm{mg} / 1 \mathrm{~mL}$ PRN. Chest X-ray, EKG and ECHO were normal with no evidence of any cardiopulmonary disease. His renal ultrasound showed no hydronephrosis, with increased echogenicity of renal cortices, suggestive of medical renal disease with a trace of lateral perinephric fluid.

He was admitted to the ward for further management. Urine output and renal plasma flow were closely monitored. Fluids were continued, titrating to $250 \mathrm{~mL} / \mathrm{h}$. The $\mathrm{CK}$ was repeated and the count dropped to 200,000 IU/L. Overnight, the patient developed stage $3 \mathrm{AKI}$ as his creatinine level started to rise from $1.33 \mathrm{mg} / \mathrm{dL}$ on admission to $7 \mathrm{mg} / \mathrm{dL}$, but his electrolytes were normal with no acid base disorder and adequate urine output.

Patient was put on renal diet with low potassium and low phosphorus with protein restriction to about $60-75 \mathrm{~g} /$ day $(0.8$ - $1 \mathrm{~g} / \mathrm{kg}$ IBW according to AKI guidelines) and also total calorie intake of about $2,250 \mathrm{kcal} / \mathrm{kg}$ (30 kcal $/ \mathrm{kg}$ IBW according to 
AKI guidelines). However, the $\mathrm{S}$. Cr continued to rise, peaking to $13.88 \mathrm{mg} / \mathrm{dL}$ on day 10 . Further blood work revealed hyperphosphatemia reaching $9.5 \mathrm{mg} / \mathrm{dL}$. As expected, calcium levels started to trend downwards from 9.4 to $6.9 \mathrm{mmol} / \mathrm{L}$. Phosphorus binder, Renagel was started. Given the young age of the patient and the permissible levels of potassium with adequate urine output, dialysis was held off and the patient was diligently monitored and treated empirically for an AKI secondary to RB.

The CK and inorganic phosphate trended downwards steadily. It is worth noting that the patient had fluctuating BP secondary to aggressive fluid therapy and AKI reaching up to $187 / 97 \mathrm{~mm} \mathrm{Hg}$ and was treated with hydralazine $10 \mathrm{mg}$ tab acutely. Efforts were made to rule out other etiologies. ANA, ANTI DNA Ds, HIV, and thyroid panel revealed negative results (Fig. 1).

The patient was counseled and discharged on request. He was discharged on famotidine $20 \mathrm{mg}$ tab, and sevelamer HCL $800 \mathrm{mg}$ and sennosides $8.6 \mathrm{mg}$ tab. On discharge, his CK level was $1,701 \mathrm{IU} / \mathrm{L}$, and phosphorus was $6.9 \mathrm{mg} / \mathrm{dL}$ with a $\mathrm{Cr}$ of $6.64 \mathrm{mg} / \mathrm{dL}$ and a BUN of $91 \mathrm{mg} / \mathrm{dL}$. His follow-up appointment with the primary and nephrologists reveal a CK of 338 $\mathrm{IU} / \mathrm{L}$ and a $\mathrm{Cr}$ of $1.99 \mathrm{mg} / \mathrm{dL}$ and the patient made a complete recovery.

\section{Discussion}

The median age of RB is 47 years; however, the incidence and risk factors for RB in younger patients have not been established [17]. A large number of risk factors are known to cause $\mathrm{RB}$ and can be categorized as intrinsic muscle dysfunction, use of prescribed medications such as amphetamines and phencyclidine hydrochloride, use of illicit drugs such as heroin, metabolic disorders, trauma, heatstroke, infections, seizure or long-term immobilization [1]. In a retrospective study of RB in young patients, Chen et al reported $87 \%$ were males with trauma $(26.7 \%)$ and infections $(18.3 \%)$ were the most common causes [18].

The association of excessive physical exertion (e.g. marathoners and military recruits) with RB is well documented in the medical literature [19]. Occurrence of RB depends on the overall fitness of the body including the targeted muscles and the intensity of physical activity performed. In 2016, Hummel et al presented a series of cases of RB in young athletes. They described numerous cases of RB caused by one or a few strenuous workout sessions after a period of prolonged inactivity [20]. This article described six cases in which patients presented with RB after participation in the CrossFit exercise regimen. These cases are significant as three of the cases involved patients that had been participating in CrossFit for months to years. This disputes the assumption that exertional RB solely occurs when exerting oneself after a prolonged period of immobility.

Often multiple confounding risk factors are present. Many cases involved the athletes admitting to poor fluid intake prior to exercising. Many athletes admitted to consuming caffeine, protein substances, and NSAIDs that may contribute to the risk of RB. Other cases involved patients suffering from heat stroke during the exercise session. In 2012, Oh et al reported on a cluster of cases of acute exertional RB precipitated by football camp resistance exercises performed in a hot building. The athletes were given little time to rehydrate, which may have led to further worsening of their condition. The authors attribute poor knowledge about risk factors and preventative strategies regarding RB [21]. Knowledge of the true prevalence of exercise-induced RB can help better educate athletic training programs. In the younger demographic population, in addition to exertional $\mathrm{RB}$, it is important to consider infection as a possible cause of RB.

Some level of CK rise is normal in exercising individuals. O'Brien et al studied serum CK levels in military recruits in training. They found that patients with exertional RB had a higher average S. CK level $(40,471 \mathrm{IU} / \mathrm{L})$ than other etiologies and had a lower incidence of AKI. They found these patients responded very well to rehydration. Despite peak serum CK levels $>100,000 \mathrm{IU} / \mathrm{L}$ in some cases, acute renal failure did not often occur, and this form of RB seemed very responsive to I/V hydration.

\section{Conclusion}

We present the case of severe RB in a young adult that occurred just after a 2-hour of workout session at the gym. His initial CPK at time of presentation was unusually high reaching over 500,000 IU/L. Despite getting aggressively bolused with IV fluids, there was rapid deterioration in kidney function and the patient advanced into AKI reaching a peak $\mathrm{Cr}$ of $13.88 \mathrm{mg} / \mathrm{dL}$. All measures were taken to closely monitoring of renal functions and CK levels. As the patient was stable with adequate urine output and no acid base disorder, we decided to treat him with IV fluids only and renal replacement therapy held off. By day 10, the S. Cr started to plateau and then decline. Our patient has made a complete recovery and to date is healthy.

Our patient's course of gradual recovery from stage 3 AKI secondary to severe $\mathrm{Rb}$ with abnormally elevated $\mathrm{CK}$ highlights the paramount importance of early IV fluids intervention and clinicians should also bear in mind the patient's overall health status before considering renal replacement therapy. More research especially in the younger population is needed to better guide clinicians in their treatment protocols.

\section{Consent}

Written informed consent was obtained from the patient for publication of this case report.

\section{References}

1. Huerta-Alardin AL, Varon J, Marik PE. Bench-to-bedside review: Rhabdomyolysis - an overview for clinicians. Crit Care. 2005;9(2):158-169. 
2. Dayer-Berenson L. Rhabdomyolysis: a comprehensive guide. ANNA J. 1994;21(1):15-18; quiz 19-20.

3. Bagley WH, Yang $\mathrm{H}$, Shah KH. Rhabdomyolysis. Intern Emerg Med. 2007;2(3):210-218.

4. Green HJ. Cation pumps in skeletal muscle: potential role in muscle fatigue. Acta Physiol Scand. 1998;162(3):201213.

5. Clausen $\mathrm{T}$. Na+-K+ pump regulation and skeletal muscle contractility. Physiol Rev. 2003;83(4):1269-1324.

6. Knochel JP. Neuromuscular manifestations of electrolyte disorders. Am J Med. 1982;72(3):521-535.

7. Knochel JP. Mechanisms of rhabdomyolysis. Curr Opin Rheumatol. 1993;5(6):725-731.

8. Guerini D, Coletto L, Carafoli E. Exporting calcium from cells. Cell Calcium. 2005;38(3-4):281-289.

9. Warren JD, Blumbergs PC, Thompson PD. Rhabdomyolysis: a review. Muscle Nerve. 2002;25(3):332-347.

10. Campanella M, Pinton P, Rizzuto R. Mitochondrial $\mathrm{Ca} 2+$ homeostasis in health and disease. Biol Res. 2004;37(4):653-660.

11. Wrogemann K, Pena SD. Mitochondrial calcium overload: A general mechanism for cell-necrosis in muscle diseases. Lancet. 1976;1(7961):672-674.

12. Rizzuto R, Pinton P, Ferrari D, Chami M, Szabadkai G, Magalhaes PJ, Di Virgilio F, et al. Calcium and apoptosis: facts and hypotheses. Oncogene. 2003;22(53):86198627.
13. Zager RA. Rhabdomyolysis and myohemoglobinuric acute renal failure. Kidney Int. 1996;49(2):314-326.

14. Rodriguez E, Soler MJ, Rap O, Barrios C, Orfila MA, Pascual J. Risk factors for acute kidney injury in severe rhabdomyolysis. PLoS One. 2013;8(12):e82992.

15. Chatzizisis YS, Misirli G, Hatzitolios AI, Giannoglou GD. The syndrome of rhabdomyolysis: complications and treatment. Eur J Intern Med. 2008;19(8):568-574.

16. Mohaupt MG. [Rhabdomyolysis]. Ther Umsch. 2003;60(7):391-397.

17. Melli G, Chaudhry V, Cornblath DR. Rhabdomyolysis: an evaluation of 475 hospitalized patients. Medicine (Baltimore). 2005;84(6):377-385.

18. Chen CY, Lin YR, Zhao LL, Yang WC, Chang YJ, Wu HP. Clinical factors in predicting acute renal failure caused by rhabdomyolysis in the ED. Am J Emerg Med. 2013;31(7):1062-1066.

19. Schiff HB, MacSearraigh ET, Kallmeyer JC. Myoglobinuria, rhabdomyolysis and marathon running. Q J Med. 1978;47(188):463-472.

20. Hummel K, Gregory A, Desai N, Diamond A. Rhabdomyolysis in adolescent athletes: review of cases. Phys Sportsmed. 2016;44(2):195-199.

21. Oh JY, Laidler M, Fiala SC, Hedberg K. Acute exertional rhabdomyolysis and triceps compartment syndrome during a high school football cAMP. Sports Health. 2012;4(1):57-62. 